\title{
Activity of the Seyfert galaxy neighbours ${ }^{\star}, \star \star$
}

\author{
E. Koulouridis ${ }^{1}$, M. Plionis ${ }^{2,3}$, V. Chavushyan ${ }^{3}$, D. Dultzin ${ }^{4}$, Y. Krongold ${ }^{4}$, I. Georgantopoulos ${ }^{1}$, and J. León-Tavares ${ }^{5,6}$ \\ ${ }^{1}$ Institute of Astronomy \& Astrophysics, National Observatory of Athens, Palaia Penteli 152 36, Athens, Greece \\ e-mail: ekoulour@noa.gr \\ 2 Physics Department of Aristotle, University of Thessaloniki, University Campus, 54124 Thessaloniki, Greece \\ 3 Instituto Nacional de Astrofísica Optica y Electrónica, Puebla, C.P. 72840 México, Mexico \\ ${ }^{4}$ Instituto de Astronomía, Universidad Nacional Autónoma de México, Apartado Postal 70-264, D. F. 04510 México, Mexico \\ 5 Finnish Centre for Astronomy with ESO (FINCA), University of Turku, Väisäläntie 20, 21500 Piikkiö, Finland \\ 6 Aalto University, Metsähovi Radio Observatory, Metsähovintie 114, 02540 Kylmälä, Finland
}

Received 15 May 2012 / Accepted 23 January 2013

\section{ABSTRACT}

\begin{abstract}
We present a follow-up study of a series of papers concerning the role of close interactions as a possible triggering mechanism of AGN activity. We have already studied the close $\left(\leq 100 h^{-1} \mathrm{kpc}\right)$ and the large-scale $\left(\leq 1 h^{-1} \mathrm{Mpc}\right)$ environment of a local sample of Sy1, Sy2, and bright IRAS galaxies (BIRG) and of their respective control samples. The results led us to the conclusion that a close encounter appears capable of activating a sequence where an absorption line galaxy (ALG) galaxy first becomes a starburst, then a Sy2, and finally a Sy1. Here we investigate the activity of neighbouring galaxies of different types of AGN, since both galaxies of an interacting pair should be affected. To this end we present the optical spectroscopy and X-ray imaging of 30 neighbouring galaxies around two local $(z \lesssim 0.034)$ samples of 10 Sy 1 and 13 Sy 2 galaxies. Although this is a pilot study of a small sample, various interesting trends have been discovered that imply physical mechanisms that may lead to different Seyfert types. Based on the optical spectroscopy, we find that more than $70 \%$ of all neighbouring galaxies exhibit star forming and/or nuclear activity (namely recent star formation and/or AGN), while an additional X-ray analysis showed that this percentage might be significantly higher. Furthermore, we find a statistically significant correlation, at a $99.9 \%$ level, between the value of the neighbour's $[\mathrm{OIII}] / \mathrm{H} \beta$ ratio and the activity type of the central active galaxy, i.e. the neighbours of Sy2 galaxies are systematically more ionized than the neighbours of Sy1s. This result, in combination with trends found using the Equivalent Width of the $\mathrm{H} \alpha$ emission line and the stellar population synthesis code STARLIGHT, indicate differences in the stellar mass, metallicity, and star formation history between the samples. Our results point towards a link between close galaxy interactions and activity and also provide more clues regarding the possible evolutionary sequence inferred by our previous studies.
\end{abstract}

Key words. galaxies: active - galaxies: Seyfert - galaxies: interactions - galaxies: nuclei - large-scale structure of Universe X-rays: galaxies

\section{Introduction}

The properties of the host galaxies of the different types of active galactic nuclei (AGN) and their environments, up to several hundred $\mathrm{kpc}$, can give us valuable information on the nature of the general AGN population, as well as on different properties of each AGN subtype. In addition, the availability nowadays of large automatically constructed galaxy catalogues, like the SDSS, can provide the necessary statistical significance for these types of analyses. However, great caution should be used when interpreting results based on large databases, since the larger the sample size, the less control one usually has on the spectral and other details of the individual galaxy entries. It could then be difficult to address important questions, such as: Do the Unification paradigm explains all cases of Type 1 and Type 2 AGN? What is the true connection between galaxy interactions, star formation, and nuclear activity? What is the lifetime of these phenomena? How do LINERs fit in the general picture,

\footnotetext{
$\star$ Appendix A is available in electronic form at http://www . aanda.org

$\star \star$ FITS files are only available at the CDS via anonymous ftp to cdsarc.u-strasbg.fr (130.79.128.5) or via

http://cdsarc.u-strasbg.fr/viz-bin/qcat?]/A+A/552/A135
}

and can all be considered AGN? Do evolutionary trends affect the AGN phenomenology?

Nowadays, it is widely accepted that the accretion of material into a massive black hole $(\mathrm{MBH})$, located at the galactic centre, is responsible for the detected excess emission (radiation not emitted by stellar photospheres) in the AGN's spectra, and these black holes do exist in all elliptical galaxies and spiral galaxy bulges (Kormendy \& Richstone 1995; Magorrian et al. 1998), including our own (e.g. Melia \& Falcke 2001). However, we still lack complete understanding of the various aspects of the nuclear activity, for example, the triggering mechanism and the feeding of the black hole, the physical properties of the accretion disk and the obscuring torus predicted by the unified scheme (Antonucci et al. 1993), the origin of jets in radio loud objects, the connection with star formation and the role of the AGN feedback. Even the exact mechanism that produces the observed infrared, X-ray, and gamma-ray emission is still only partially understood (e.g. León-Tavares et al. 2011). Although successful in many cases, the unification model has not been able to fully explain all the AGN phenomenology (among others, the role of interactions on induced activity; Koulouridis et al. 2006a,b, and references therein).

Despite observational difficulties and limitations, there have been many attempts, based on different diagnostics, to 
investigate the possible triggering mechanisms of nuclear activity. Most agree that the accretion of material into a $\mathrm{MBH}$ (Lynden-Bell 1969) is the mechanism responsible for the emission, but it is still necessary to understand the feeding mechanism of the black hole. It is known and widely accepted that interactions between galaxies can force gas and molecular clouds towards the galactic centre, where they become compressed and produce starburst events. (e.g., Li et al. 2008; Ellison et al. 2008; Ideue et al. 2012). Many also believe that the same mechanism could give birth to an active nucleus (e.g. Umemura 1998; Kawakatu et al. 2006; Ellison et al. 2011; Silverman et al. 2011; Villforth et al. 2012). Even though the exact mechanism is still unknown, in the local Universe a minimum accretion rate of $\sim 10^{-6 \pm 1} M_{\odot} / \mathrm{yr}$ is needed to fuel the black hole (Ho 2008). At such low accretion rates, nuclear activity is probably relatively weak compared to the host galaxy, and most of the spectral signatures of the AGN are "buried". Theoretically the feeding of the black hole can only be achieved by means of a non-axisymmetric perturbation that induces mass inflow. This kind of perturbations can be provided by interactions, and the result of the inflow is feeding of the black hole and activation of the AGN phase, maybe $\sim 50-250 \mathrm{Myr}$ after the initial interaction took place (see below). An interaction certainly predicts such a time delay, since after the material has piled up around the inner Linblad resonance, enhancing star formation, it can be channelled towards the nucleus by loosing significant amounts of angular momentum, a process that is not instantaneous.

Indeed, post starburst stellar populations have been observed around AGN (Dultzin-Hacyan \& Benitez 1994; Maiolino \& Rieke 1995; Nelson \& Whittle 1996; Hunt et al. 1997; Maiolino et al. 1997; Boisson et al. 2000, 2004; Cid Fernandes et al. 1998, $2001,2004,2005)$ and in close proximity to the core $(\sim 50 \mathrm{pc})$. This fact implies the continuity of these two states and a delay of 50-250 Myr between the onset of the starburst and the feeding of the AGN (e.g., Müller Sánchez et al. 2008; Wild et al. 2010; Davies et al. 2012), which may reach the peak of its activity after $\sim 500 \mathrm{Myr}$ (Kaviraj et al. 2011). When studying the cosmic X-ray background (CXRB), Ballantyne et al. (2006) conclude that Seyfert galaxies (dominating in the production of the CXRB) are likely fueled by minor mergers or interactions that can trigger a circumnuclear star formation event, but that there may be a significant delay between the interaction and the ignition of the nucleus. After analyzing star formation in the nuclei of nine Seyfert galaxies, Davies et al. (2007) found recent, but no longer active, starbursts that occurred 10-300 Myr ago. Further support for an interaction-activity relation has recently been provided by HI observations of Tang et al. (2008), who found that $94 \%$ of the Seyfert galaxies in their sample were disturbed in contrast to their control sample (where only $19 \%$ were disturbed), but see also Georgakakis et al. (2009) and Cisternas et al. (2011) in the AEGIS and cosmos surveys, respectively.

This paper is the third in a series of three-dimensional studies of the environment of active galaxies (Koulouridis et al. 2006a,b), extending previous 2D analyses (Dultzin et al. 1999; Krongold et al. 2002) in an effort to shed more light on the starburst/AGN connection and on the evolutionary scenario, triggered by interactions, proposed in our previous papers. It is a follow-up spectroscopic pilot study aiming at investigating the possible effects of interactions on the neighbours of our Seyfert galaxies and understanding the conditions necessary for the different types of activity.

In Sect. 2 we discuss our galaxy samples and present our observations and data reduction. The spectroscopic analysis and classification of the galaxies, basic host galaxy properties, results from STARLIGHT stellar population synthesis code, and the analysis of the available X-ray observations are presented in Sect. 3. Finally, in Sect. 4 we will interpret our results and draw our conclusions. All distances are calculated taking the local velocity field into account (which includes the effects of the following structures: Virgo, Great Attractor and Shapley) for the standard $\Lambda$ CDM cosmology $\left(\Omega_{\mathrm{m}}=0.27, \Omega_{\Lambda}=0.73\right)$. Throughout our paper we use $H_{\circ}=100 \mathrm{hm} \mathrm{s}^{-1} \mathrm{Mpc}^{-1}$, following our previous study of the same samples.

\section{Data}

\subsection{Sample definition and previous results}

The samples of active galaxies were initially compiled from the catalogue of Lipovetskij et al. (1987), which itself is a compilation of all Seyfert galaxies known at the time from various surveys and in various frequencies (optical, X-ray, radio, infrared). It includes all extended objects and several starlike objects with absolute magnitudes lower than -24 . Available multi-frequency data are listed, including: coordinates, redshifts, Seyfert type (and sub-type), $U B V R$-photoelectric magnitudes, morphological types, fluxes in $\mathrm{H} \beta$ and [OIII]5007, JHKLN fluxes, far-infrared (IRAS) fluxes, radio fluxes at 6 and $11 \mathrm{~cm}$, monochromatic $\mathrm{X}$-Ray fluxes in $0.3-3.5$ and $2-10 \mathrm{keV}^{1}$. About half of the listed Seyfert galaxies can also be found in the IRAS catalogue.

Dultzin-Hacyan et al. (1999) selected from the catalogue two volume-limited and complete samples, consisting of $72 \mathrm{Sy} 1$ and 60 Sy2, to study their projected circumgalactic environment. In Koulouridis et al. (2006a) we used practically the same samples to verify their results, using in addition redshift data from the CFA2 and SSRS surveys and our own deeper spectroscopic observations. Well-selected control samples (same redshift, diameter, and morphology distributions) were used for the comparison in both studies.

Using the CfA2 and SSRS redshift catalogues, and our own deeper low-resolution spectroscopic observations (reaching to $m_{B} \sim 18.5$ ), we searched for neighbours within a projected distance $R \leq 100 h^{-1} \mathrm{kpc}$ and a radial velocity separation $\delta u \leq$ $600 \mathrm{~km} \mathrm{~s}^{-1}$ and we found that:

- The Sy1 galaxies and their control sample show a similar (consistent within $1 \sigma$ Poisson uncertainty) fraction of objects having at least one close neighbour.

- There is a significantly higher fraction of Sy2 galaxies having a near neighbour, especially within $D \leq 75 h^{-1} \mathrm{kpc}$, compared to both their control sample and the Sy1 galaxies.

- The large-scale environment of Sy 1 galaxies $\left(D=1 h^{-1} \mathrm{Mpc}\right.$ and $\delta u \leq 1000 \mathrm{~km} \mathrm{~s}^{-1}$ ) is denser than that of Sy 2 galaxies, although it is consistent with their respective control samples.

- Using deeper spectroscopic observations of the neighbours for a random subsample of 22 Sy 1 and 22 Sy 2 galaxies we found that the differences between the close environment of Sy1 and Sy2's persists even when going to fainter neighbours, correspond to a magnitude similar to the one of the Large Magellanic Cloud.

For the purposes of the present study, we obtained new mediumresolution spectroscopy to resolve the $\mathrm{H} \alpha$ and [NII] lines - unresolved in our original low-resolution spectra, of all the neighbours around the aforementioned subsamples of the 22 Sy1

1 All data can be found online at the vizier database (http:// vizier. cfa.harvard. edu/viz-bin/VizieR?-source=VII/ 173). 
and 22 Sy2, respectively. In Tables 1 and 2 we present the names, celestial coordinates, $O_{\text {MAPS }}$ magnitudes ${ }^{2}$, and redshifts of the Sy1 and Sy2 galaxies that have at least one close neighbour (within $\delta u<600 \mathrm{~km} \mathrm{~s}^{-1}$ ). The full samples are presented in detail in Koulouridis et al. (2006a,b). We have kept the original neighbours enumeration of the previous papers (for example, in Table 2, NGC 1358 has only neighbour 2, since neighbour 1 had $\delta u>600 \mathrm{~km} \mathrm{~s}^{-1}$ ).

\subsection{Spectroscopic observations}

We have obtained medium-resolution spectroscopic data of all the neighbouring galaxies in our samples in order to classify them according to their optical emission lines (Sect. 2.3). Optical spectra were taken with the Boller \& Chivens spectrograph mounted on the $2.1 \mathrm{~m}$ telescope at the Observatorio Astronómico Nacional in San Pedro Mártir (OAN-SPM). Observations were carried out during photometric conditions. All spectra were obtained with a 2'.5 slit. The typical wavelength range was 4000-8000 $\AA$ and the spectral resolution $R=8 \AA$. Spectrophotometric standard stars were observed every night.

The data reduction was carried out with the IRAF $^{3}$ package following a standard procedure. Spectra were biassubtracted and corrected with dome flat-field frames. Arc-lamp (CuHeNeAr) exposures were used for wavelength calibration. All emission line galaxy spectra can be found in Appendix A.

\subsection{Analysis and classification method}

In this section we present the results of our spectroscopic observations of all the neighbours with $D \leq 100 h^{-1} \mathrm{kpc}$ and $m_{O_{\text {MAPS }}} \lesssim 18.5$ for the samples of Sy 1 and Sy 2 galaxies. We also used SDSS spectra when available.

Our aim was to measure six emission lines: $\mathrm{H} \beta \quad \lambda 4861$,

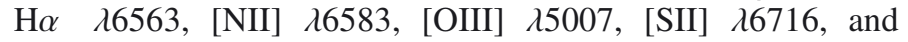
[SII] $\lambda 6731$, in order to classify our galaxies, using the Baldwin et al. (1981, hereafter BPT), and Veilleux \& Osterbrock (1987) diagrams. For the cases where it was not possible to measure the $\mathrm{H} \beta$ and [OIII] emission lines, we use the more approximate classification by Stasińska et al. (2006).

Based on the above, we adopted the following classification scheme:

- absorption line galaxies (ALG), i.e. galaxies with no emission lines;

- galaxies with emission lines (ELG), meaning that they exhibit nuclear or/and recent star forming activity.

Flux ratios for the emission lines mentioned above have been measured after subtracting the host galaxy contamination from each spectrum. We disentangled the spectral contribution of the host galaxy from the observed spectra by using the stellar population synthesis code STARLIGHT ${ }^{4}$. Spectra processing and fits were carried in the same fashion as described in Sect. 3.1 of León-Tavares et al. (2011). For a detailed description of

\footnotetext{
$2 O$ (blue) POSS I plate magnitudes of the Minnesota Automated Plate Scanner (MAPS) system. We used $O_{\text {MAPS }}$ magnitudes because Zwicky magnitudes were not available for the fainter neighbours, and we needed a homogeneous magnitude system for all our objects.

3 IRAF is distributed by National Optical Astronomy Observatories operated by the Association of Universities for Research in Astronomy, Inc. under cooperative agreement with the National Science Foundation.

4 http://starlight.ufsc.br/
}

the STARLIGHT code and its scientific results, we refer to the papers of the SEAGal collaboration (Mateus et al. 2006; Asari et al. 2007; Cid-Fernandes et al. 2005, 2007). We only note that we have calculated the $1 \sigma$ standard deviation of the flux as follows (Tresse et al. 1999):

$$
\sigma=\sigma_{\mathrm{c}} d \sqrt{2 N_{\text {pix }}+E W / d}
$$

where $\sigma_{\mathrm{c}}$ is the standard deviation of the continuum about the emission line, $d$ is the spectral dispersion in $\AA$ per pixel and $N_{\text {pix }}$ is the base-width of the emission line in pixels. In our case the parameter $d \sim 4 \AA /$ pix, while for the SDSS spectra is $\sim 1.1 \AA /$ pix for the $\mathrm{H} \beta$ area and $\sim 1.5 \AA$ /pix for the $\mathrm{H} \alpha$ area. To the above we have added in quadrature the errors of the Gaussian fitting of the emission lines. We should note here that in some cases the $B$ telluric band is very close to the [SII] doublet (see for example NGC 1019-N2 on the left of the doublet or UGC 7064-N1B on the right of the doublet) introducing a further uncertainty on the calculation of the flux. In all these cases, we have simultaneously fitted the telluric absorption and the emission lines to better measure the $[\mathrm{SII}]$ doublet's flux. Although we do not have any exact evaluation of the uncertainty due to the above spectral feature, we presume (at least for the cases that the $B$ telluric band is close to the doublet) that the reported error is underestimated ${ }^{5}$.

Although it is possible to distinguish between a star forming nucleus (SFN) galaxy ${ }^{6}$ and an AGN using only the $[\mathrm{NII}] / \mathrm{H} \alpha$ ratio, we cannot distinguish between a low-ionization (LINER) and a high ionization (Seyfert) AGN galaxy. We have also measured $[\mathrm{OI}](\lambda=6300)$ when possible, as an extra indicator of AGN activity. However, the weakness of the line in most cases did not allow further use of it in a separate BPT diagram.

In Fig. 1a we plot the line ratios $\log ([\mathrm{OIII}] / \mathrm{H} \beta)$ versus $\log ([\mathrm{NII}] / \mathrm{H} \alpha)$ (BPT diagram) for those neighbours of Seyfert galaxies for which we have the four necessary emission lines available ${ }^{7}$. We also plot the Kauffmann et al. (2003a) separation line between SFN and AGN galaxies, given by

$$
\log ([\mathrm{OIII}] / \mathrm{H} \beta)=\frac{0.61}{(\log ([\mathrm{NII}] / \mathrm{H} \alpha)-0.05}+1.3
$$

and the corresponding one of Kewley et al. (2001):

$$
\log ([\mathrm{OIII}] / \mathrm{H} \beta)=\frac{0.61}{(\log ([\mathrm{NII}] / \mathrm{H} \alpha)-0.47}+1.19 .
$$

We also plot in Fig. $1 \mathrm{~b}$ the line ratios $\log ([\mathrm{OIII}] / \mathrm{H} \beta)$ vs. $\log ([\mathrm{SII}] / \mathrm{H} \alpha)$. Qualitatively, the same results as those presented in Fig. 1a are repeated here as well. The dividing line is given by Kewley et al. (2006a,b). However, we do not have the respective line of Kauffmann et al. (2003a), as it is not available in the literature, and thus we cannot separate pure star forming galaxies from composite objects. Since, as already discussed, the measurement of the [SII] doublet is probably contaminated by absorption of the $B$ telluric band, we present only results based on the $[\mathrm{NII}]$ forbidden line.

\footnotetext{
5 We should also note that the standard deviation of the continuum about the [SII] doublet was calculated after the subtraction of the $B$ telluric band.

6 We choose to call SFN all galaxies with prominent emission lines that do not show AGN activity.

7 We have excluded one merger neighbour (UGC 7064-N1) since its two nuclei are in an advanced merging state and their properties are most probably independent of any interaction that may have with the central active galaxy.
} 


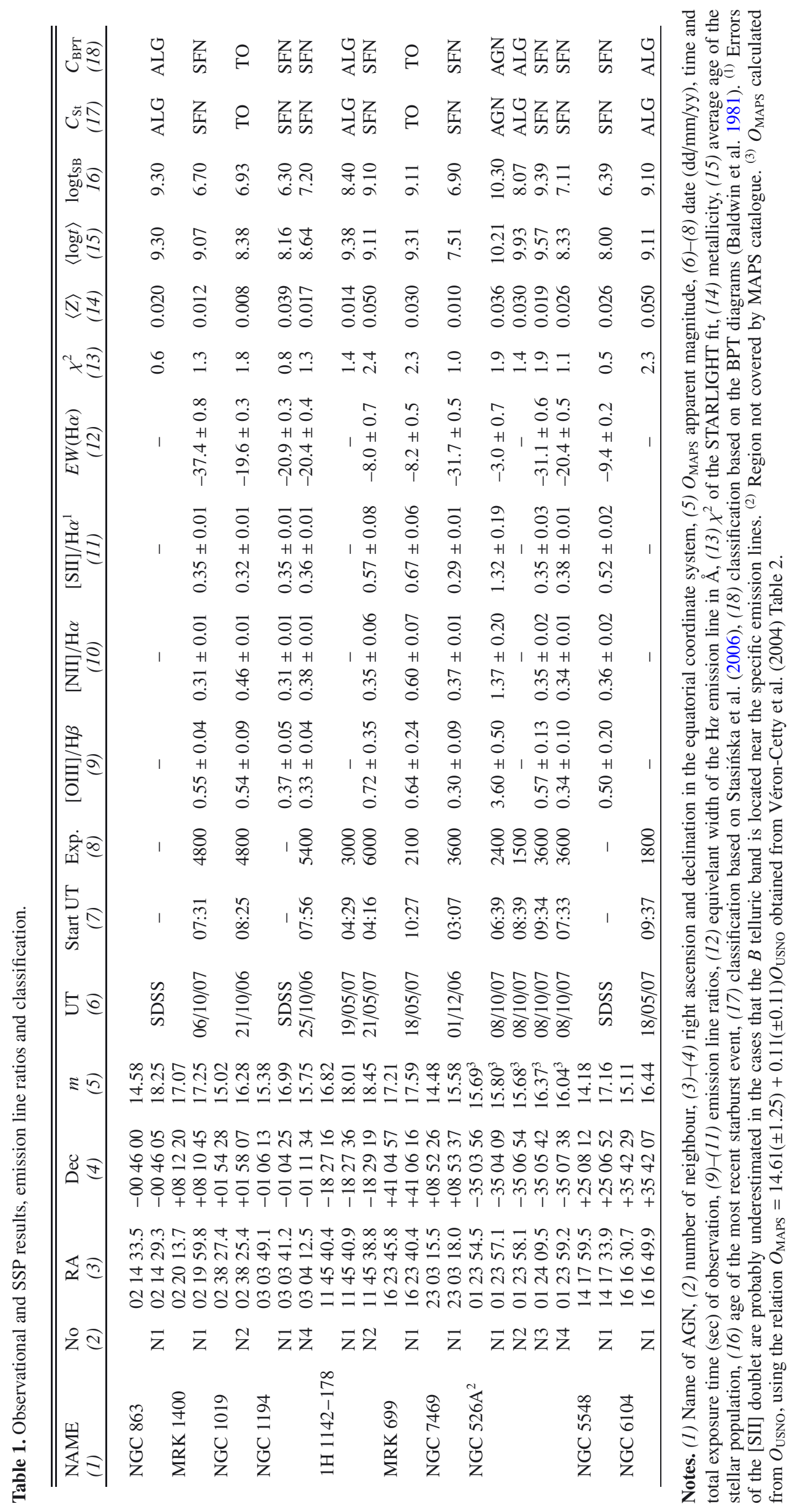


E. Koulouridis et al.: Neighbours of Seyfert galaxies

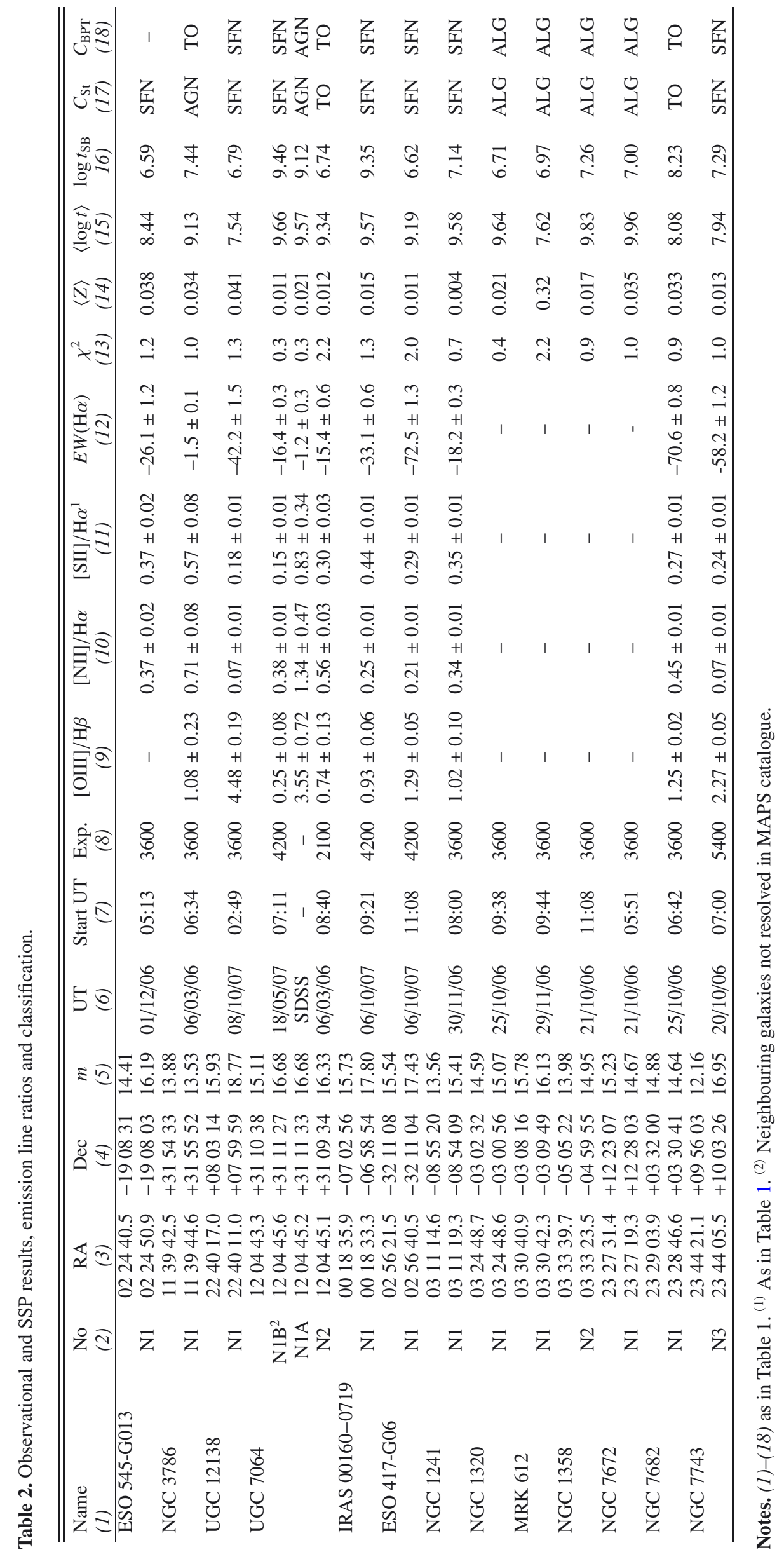



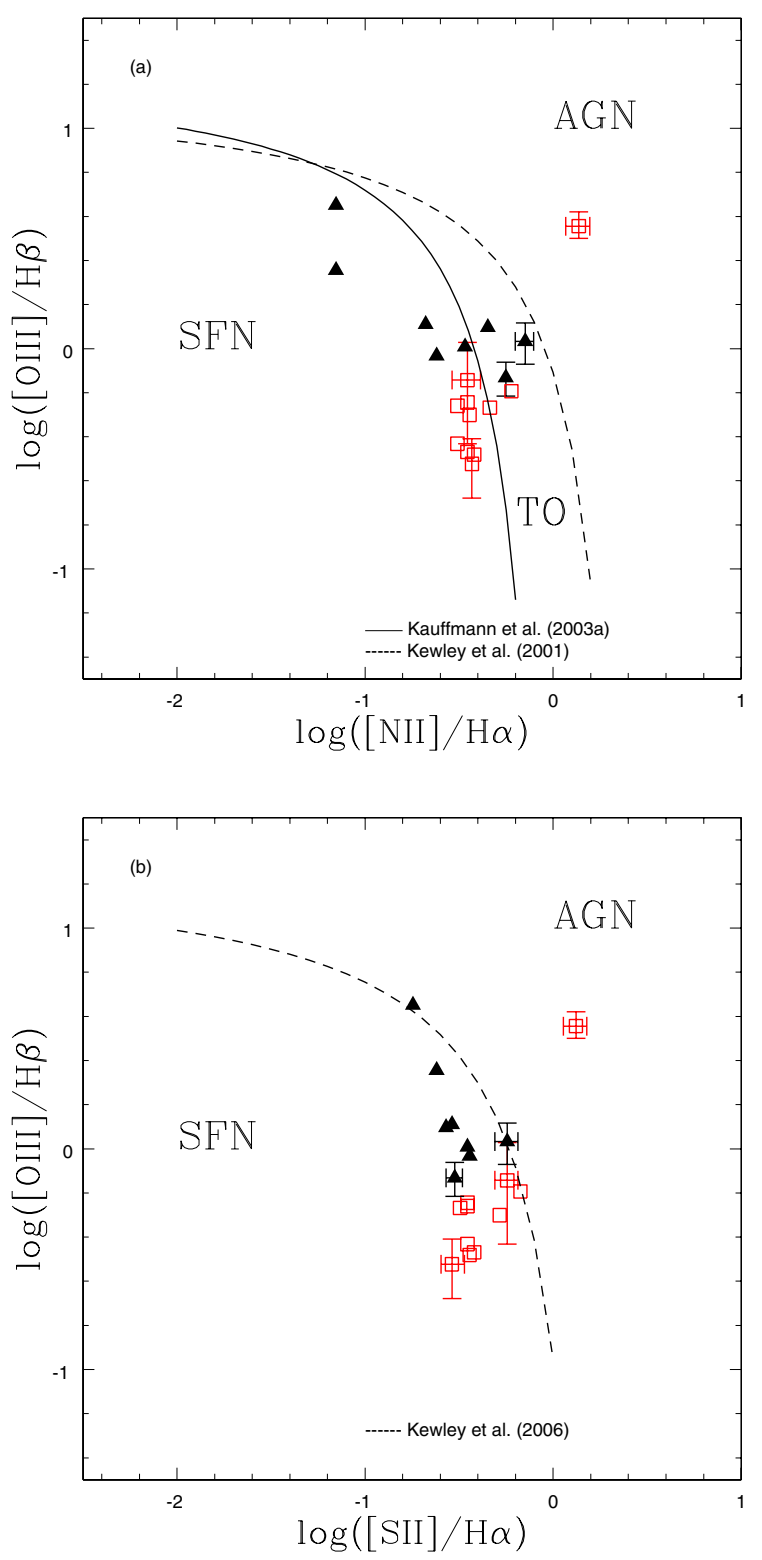

Fig. 1. a) BPT diagram, and b) Veilleux \& Osterbrock classification diagram of the neighbours of Sy1 and Sy2 galaxies. The neighbours of Sy2 and Sy1 galaxies are indicated by black triangles and red squares, respectively. For clarity, only the errorbars for those galaxies with the largest uncertainties in the $[\mathrm{OIII}] / \mathrm{H} \beta$ ratio are presented in the diagrams.

We can now classify our objects in the following categories:

- SFN: all the objects that are found below the line of Kaufmann et al.

- AGN: the objects found above the line of Kewley et al.

- TO (transition object): the ones that are found between the two lines and exhibit characteristics of both nuclear activity and recent star formation.

We do not attempt to divide the star forming galaxies into more subcategories since such a categorization appears to be highly subjective and depends on the applied methodology (e.g. Knapen \& James 2009).

For one of our objects (ESO 545-G013-N1), the $\mathrm{H} \beta$ and [OIII] ( $\lambda 5007)$ lines were not observed, so we classified it using the more approximate method of Stasińska et al. (2006), which is based solely on the NII/H $\alpha$ ratio. in order to evaluate this method, we applied it to all our galaxies and found a consistency with the BPT classification in all cases but one (see Table 3).

Further classification of the Seyfert galaxies in Types 1 and 2 was obtained by direct visual examination of the spectra from the broadening of the emission lines. No broad lines were discovered in the spectrum of the two neighbours classified as AGN and therefore they should be considered as Type 2. In Tables 1 and 2 we list, for all neighbours, their line ratios and the two different classifications.

We also measured the equivalent width of the $\mathrm{H} \alpha$ emission line, in order to use it as an extra indicator of the galaxy's star forming history, in addition to the STARLIGHT code's results. The minimum equivalent width, defined as the integrated local continuum rms noise normalized to the level of the local continuum, at a $5 \sigma$ confidence level, is found to be $E W_{\min } \sim 1 \AA$. We should note here that in a small number of cases the [OIII] and $\mathrm{H} \beta$ lines were detected only after the subtraction of the continuum. We calculated the $1 \sigma$ standard deviation of the $\mathrm{EW}$ as follows (Tresse et al. 1999):

$\sigma_{\mathrm{EW}}=\frac{E W}{F} \sigma_{\mathrm{c}} d \sqrt{2 N_{\text {pix }}+E W / d+(E W / d)^{2} / N_{\text {pix }}}$

where $\sigma_{\mathrm{c}}$ is the standard deviation of the continuum about the emission line, $d$ is the spectral dispersion in $\AA$ per pixel, $N_{\text {pix }}$ is the base width of the emission line in pixels and $F$ the flux of the emission line.

\section{Results and analysis}

\subsection{Activity of the neighbours}

In this section we discuss the results of our spectroscopy and classification in more detail. We have excluded the merging neighbour of UGC 7064, since the properties of its two nuclei are more affected by their mutual interaction rather than by their neighbouring Seyfert. We can draw our first results for each sample separately inspecting Tables 1 and 2. From the analysed 15 neighbours of Sy1 only 4 are ALGs, while 8 of them are SFNs, 2 are classified as TOs, and one is classified as AGN. Similar results hold for the neighbours of Sy2 galaxies. 4 out of 13 neighbours do not present emission lines, 6 are SFNs, and 3 are TOs. Therefore, at least $70 \%$ of the neighbours, within $100 h^{-1} \mathrm{kpc}$, of both type of Seyfert galaxies have emission lines. We should note here that Ho et al. (1997), studying a magnitude limited sample of galaxies $\left(B_{T} \leq 12.5\right)$, came up with a similar high percentage of activity $(86 \%)$. However, the results of our sample of faint neighbours cannot be directly compared with those of Ho et al. due to the brighter magnitude limit of the latter.

We can extract one of the most interesting results of our analysis by examining Fig. 1, i.e., that the neighbours of Sy 2 galaxies have systematically higher values of $[\mathrm{OIII}] / \mathrm{H} \beta$ than the neighbours of Sy 1 galaxies. Using a Kolmogorov-Smirnov twosample test for the $[\mathrm{OIII}] / \mathrm{H} \beta$ ratio we find that the null hypothesis that the samples are drawn from the same parent population is rejected at a $99.9 \%$ level. Especially for those galaxies that exhibit only star formation, the ratio [OIII]/H $\alpha$ is mainly related to their ionization level. This fact could indicate a more recent starburst event in the neighbours of Sy2 galaxies than of Sy1's, caused possibly by the interaction with a neighbouring galaxy, 
E. Koulouridis et al.: Neighbours of Seyfert galaxies

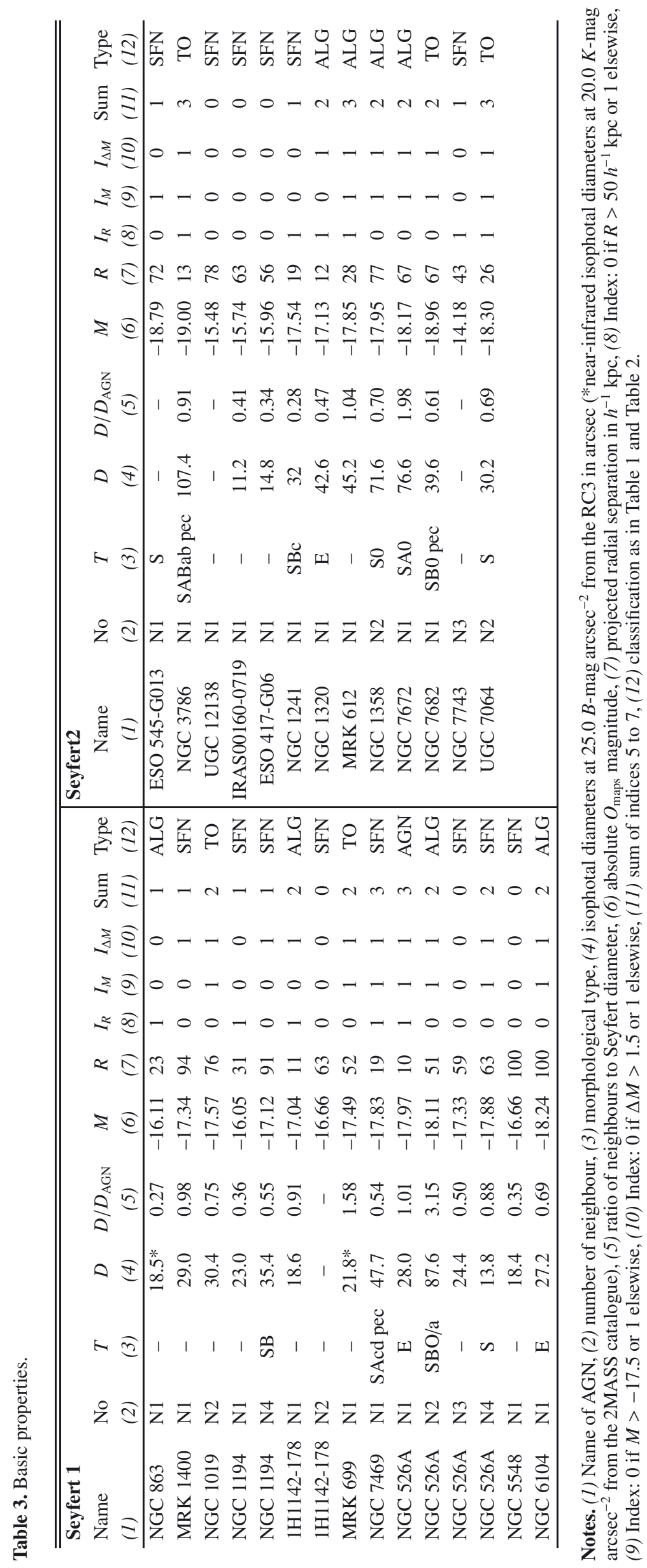


or an effect of the galaxy downsizing; i.e., more massive galaxies have formed their stellar populations earlier than less massive ones. Asari et al. (2007) argue that the location of galaxies on the BPT diagram is considered to be a result of downsizing. Should the downsizing explanation be true, the ionization level can be considered as an indicator of metallicity, which is closely related to the stellar mass. Thus, galaxies having lower values of $[\mathrm{OIII}] / \mathrm{H} \beta$ would be more massive and would have higher metallicities, indicative of an older average age of the stellar population. In Tables 1 and 2 we can see a weak trend of the mean stellar metallicity $(\langle Z\rangle)$ values (extracted from STARLIGHT) for the Sy2 SFN neighbours being lower compared to that of the Sy1's and although stellar masses cannot be directly derived from our data, most low metallicity SFNs are also faint and small in size (Table 3). However, no trend can be found by comparing the average age of the stellar populations, and given the small number of galaxies these results remain rather inconclusive.

The equivalent width of the $\mathrm{H} \alpha$ emission line is also a good indicator of the star formation history, since it represents the ratio of present to past star formation; i.e., during a starburst event young massive stars strengthen the emission lines and enhance their EW, but as time passes the strength of the emission line fades, the continuum rises again and the value of the EW declines. The highest values of the $\operatorname{EW}(\mathrm{H} \alpha)$ can be found in the spectra of the star forming neighbours of our Sy2 sample, while on the other hand some of the lowest values can be found in the respective Sy1's neighbours spectra.

A more direct way to explore the possibility that the differences of the ionization level is due to the age of the interaction of the central active galaxies with its neighbour, is by determining the age of the most recent peak of star formation with the STARLIGHT code. As it was expected, however, most of the star forming galaxies present a recent event within the last $20 \mathrm{Myr}$, a necessary fact in order to detect strong emission lines, and we can not detect any significant differences between Sy1 and Sy2 SFN neighbours. On the other hand, an interesting result is the fact that six out of seven Sy2's non-SFN (ALG, AGN, or TO) companions present a recent star formation peak $<30 \mathrm{Myr}$, while six out seven Sy1's corresponding neighbours are "quiet" for more than 100 Myr. The above fact may indicate that indeed the Sy1 galaxies have interacted with their neighbour earlier than the Sy2s.

Summarizing our main results of this section:

- More than $70 \%$ of the neighbours of the two AGN samples exhibit optical emission lines, indicating recent star formation and/or nuclear activity.

- Around $30 \%$ of the neighbours of Sy1 and Sy 2 galaxies show the presence of AGN activity, mainly in the form of TOs.

- The neighbours of Sy2s are systematically more ionized than the neighbours of Sy1s and their $\mathrm{EW}(\mathrm{H} \alpha)$ values tend also to be higher.

- Most of the non-SFN neighbours of Sy2 galaxies show a recent starburst event ( $<30 \mathrm{Myr}$ ), while the corresponding age for most of the Syl's neighbours is $>100$ Myr.

- The previous two results indicate differences in the star formation history of the neighbours of different types of AGN as well as in the age of the most recent interaction.

Finally we should note how close to a composite state are the neighbours of active galaxies, in agreement with Kewley et al. (2006a) who showed that the star forming members of close pairs, lie closer to the classification line than the star forming field galaxies. We suggest that galaxies between the curves of
Kauffmann et al. (2003) and Kewley et al. (2001) possibly migrate from a pure star forming phase to a pure AGN phase. This suggestion is very important to the formulation of a possible evolutionary scenario and is also discussed further in Sect. 4.

\subsection{Magnitude and distance analysis}

Since we have already applied a homogeneous magnitude system to our samples, we can now study whether there is a correlation between the activity of an interacting pair of galaxies and their magnitudes. The activity-magnitude comparison is performed by examining the absolute magnitude difference between the neighbour and the central active galaxy $(\Delta M)$, with low values $(\Delta M<1.5)$ indicating a stronger pair interactions. We tag these pairs as equally bright. Another parameter that can be used is the absolute magnitude of the neighbour, indicating its size. On average, absolute magnitude and size are correlated in small redshift intervals (as it is in our case), and therefore we can safely presume that a faint galaxy is also small in size and a bright one is large. The latter has been also optically inspected for our galaxies to further confirm the correlation (see also maps of Fig. 3), while the median absolute magnitude $M=-17.49$ is considered to be the separating limit between bright and faint companions. In addition we also examine the isophotal diameters at 25.0 B-mag $\operatorname{arcsec}^{-2}$ ) from the Third Reference Catalogue of bright galaxies (RC3) to compare with the absolute magnitudes, by considering any neighbour with $D / D_{\mathrm{AGN}}<1 / 2$ as being small. In two cases, because of lack of RC3 data, their near-infrared isophotal diameters (at $20.0 \mathrm{~K}$-mag $\mathrm{arcsec}^{-2}$ ) from the Two Micron All Sky Survey (2MASS) extended object catalogue were used for the comparison. We should note that only in the case of NGC 1241 the diameter criterion does not agree with the absolute magnitude criterion (marginally) and by also inspecting the SDSS image we concluded that the neighbour is indeed small. Finally, radial separation can also be considered as a crucial factor of the strength of the interaction. In Table 3 we list all the above-mentioned values plus three respective indices than take values between 0 and 1 . With 1 we denote a value that is in favour of the interaction, with 0 the opposite. In more detail, if the radial separation $R$ is less than $50 h^{-1} \mathrm{kpc}$ the respective in$\operatorname{dex} I_{D}$ is 1 and the same is true for bright neighbours and equally bright pairs, since all these factors may affect positively the interactions between two galaxies. The sum of the three indices is also listed in Table 3. Obviously the strength of the interaction of a neighbour with the sum of the three indices equal to 0 (i.e. small and faraway neighbour of a large AGN) would be significantly different from one with a sum equal to 3 (i.e. large and close galaxy of a comparable sized AGN). It therefore becomes evident that

- All faint neighbours and all neighbours of a non-equally bright pair of galaxies are primarily absorption line or purely SFN.

- All neighbours that host an AGN or are transition objects (TO), fall in the bright category, and are neighbours of an equally bright pair.

- All neighbours with interaction indices sim $\leq 1$ are purely star forming galaxies.

- All ALGs, AGN, and TO galaxies have interaction indices sum $\geq 2$ (except NGC 863-N1).

From our results we can infer that when a faint/small galaxy comes in interaction with another galaxy, the encounter induces at most a starburst but no AGN activity in the small galaxy; 
Table 4. XMM-Newton observations.

\begin{tabular}{lccccccc}
\hline \hline Name & Neigh. No & 2XMM ID & Opt. Class & $\begin{array}{c}\log L_{\mathrm{X}}(0.2-12 \mathrm{keV}) \\
\left(\mathrm{erg} \mathrm{s}^{-1}\right)\end{array}$ & $\begin{array}{c}\text { Flux (0.2-12 keV) } \\
\left(\mathrm{erg} \mathrm{cm}^{-2} \mathrm{~s}^{-1}\right)\end{array}$ & $\begin{array}{c}\text { X/O offset } \\
(\mathrm{arcmin})\end{array}$ \\
\hline NGC 1194 & N1 & - & SFN & $<39.59$ & $<7.3 \times 10^{-15}$ & - & $\begin{array}{c}\text { HR } \\
<1.1 \times 10^{-14}\end{array}$ \\
NGC 1194 & N4 & - & SFN & $<39.72$ & - & - \\
NGC 526A & N1 & J012357.0-350410 & AGN & 40.46 & $3.3 \times 10^{-14}$ & 0.023 & $-0.28 \pm 0.09$ \\
NGC 526A & N2 & J012358.1-350653 & ALG & 40.75 & $5.9 \times 10^{-14}$ & 0.008 & $0.05 \pm 0.1$ \\
NGC 526A & N3 & - & SFN & $<39.65$ & $<4.7 \times 10^{-15}$ & - & - \\
NGC 526A & N4 & J012359.0-350741 & SFN & 39.95 & $9.4 \times 10^{-15}$ & 0.035 & $-0.61 \pm 0.29$ \\
UGC 12138 & N1 & - & SFN & $<40.63$ & $<2.8 \times 10^{-14}$ & - & - \\
NGC 1320 & N1 & J032448.6-030057 & ALG & 39.46 & $1.2 \times 10^{-14}$ & 0.020 & $-0.38 \pm 0.17$ \\
MRK 612 & N1 & J033042.5-030949 & ALG & 39.59 & $3.4 \times 10^{-15}$ & 0.060 & $-0.67 \pm 0.24$ \\
NGC 1358 & N2 & J033323.3-045953 & ALG & 40.19 & $3.8 \times 10^{-14}$ & 0.044 & $0.05 \pm 0.3$ \\
NGC 7682 & N1 & J232846.7+033041 & TO & 42.04 & $1.30 \times 10^{-12}$ & 0.026 & $-0.32 \pm 0.02$ \\
NGC 7743 & N3 & - & SFN & $<39.44$ & $3.4 \times 10^{-14}$ & - \\
NGC 3786 & N1 & J113944.3+315547 & TO & 39.73 & $2.7 \times 10^{-14}$ & 0.08 & $-0.46 \pm 0.30$ \\
\hline
\end{tabular}

however it can trigger a bright AGN in the larger one. This could be due to the absence in small galaxies of a massive black hole (Wang \& Kauffmann 2007; Volonteri et al. 2008). If this assertion is correct, only galaxies that experience a major close interaction or merger can exhibit AGN activity, and this could be why AGN hosts are more frequently found in early type galaxies (e.g., Marquez \& Moles 1994; Moles et al. 1995; Ho et al. 1997; Knapen et al. 2000; Wake et al. 2004). This can also account for the large fraction of star forming galaxies among our samples of neighbours.

To cover all aspects of this issue, we should mention here that Galaz et al. (2011) show that the fraction of low surface brightness galaxies hosting an AGN is significantly lower than the corresponding fraction of high surface brightness galaxies, independently of the mass. So the deficiency of AGN in faint galaxies seems to be due to an intrinsic inability of these galaxies to host or to feed a massive black hole.

Our results indicate that the interaction of a bright galaxy especially in an equally bright pair results in an AGN or an ALG. Finding some massive galaxies, members of an equally bright interacting pair, without emission lines implies either a noneventful interaction or a delay of the outcome of the interaction. On the other hand, weak star formation or low luminosity nuclear activity may not be detectable by optical spectroscopy, although it could possibly be detected in X-rays. Such an analysis is presented below.

\subsection{The XMM-Newton observations}

We explore here, using the XMM-Newton public archive, whether the neighbours show X-ray activity. We find that 13 target fields have been observed by XMM-Newton. However, some of them are very bright and have been observed in partial window mode, rendering the observations in centre of the field-ofview unusable (NGC 5548, NGC 863, 1H1142-178, NGC 7469). The list of the remaining observations (13 neighbours and 9 central Seyfert galaxies) is shown in Table 4, in which we present $\mathrm{X}$-ray fluxes for the detections, as well as upper limits for the undetected sources. The fluxes have been taken from the 2XMM catalogue (Watson et al. 2009). The fluxes refer to the total $0.2-12 \mathrm{keV}$ band for the PN detector or the combined MOS detectors in the case where PN fluxes are not available

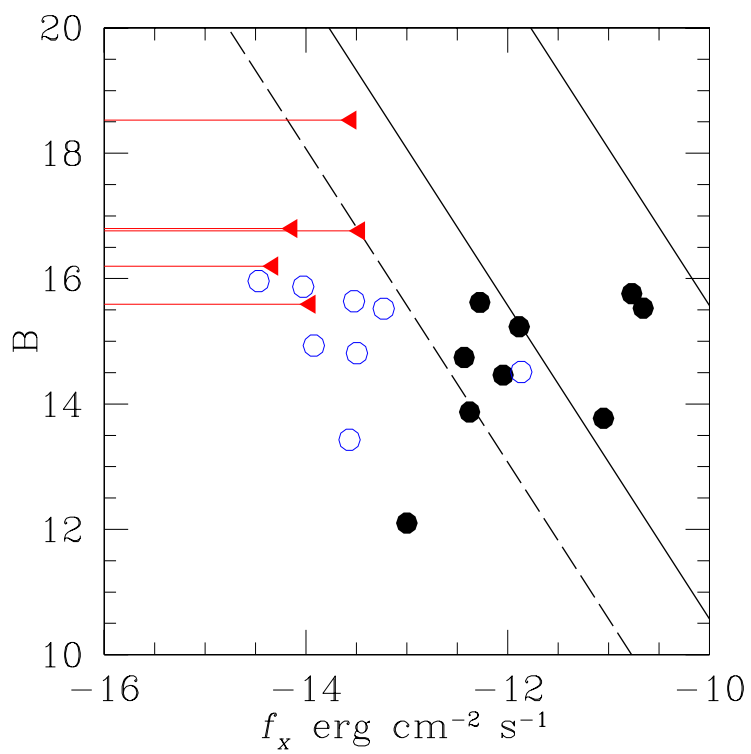

Fig. 2. X-ray (0.2-12 keV) to optical ( $B$-band) flux diagram for both the central active galaxy targets (solid circles) and the neighbours (open circles). The triangles (upper limits) denote the neighbours with no X-ray detection. The upper, lower solid line, and the dashed line correspond to $f_{\mathrm{X}} / f_{\mathrm{B}}=+1,-1,-2$ respectively. The only neighbour (open circle) that lies in the AGN regime is NGC 7682-N1.

and are estimated using a photon index of $\Gamma=1.7$ and an average Galactic column density of $N_{\mathrm{H}}=3 \times 10^{20} \mathrm{~cm}^{-2}$. Luminosities were estimated using the same spectral parameters. In the same table we quote the 2XMM hardness ratios, derived from the $1-2 \mathrm{keV}$ and $2-4.5 \mathrm{keV}$ bands (hardness ratio-3 according to the 2XMM catalogue notation). The upper limits, derived using the FLIX software, are estimated following the method of Carrera et al. (2007). This provides upper limits to the X-ray flux at a given point in the sky covered by XMM-Newton pointings. The radius used for deriving the upper limit was 20 or 30 arcsec depending on the presence of contaminating nearby sources.

In Fig. 2 we present the X-ray to optical flux diagram $f_{\mathrm{X}}-f_{\mathrm{B}}$ (e.g. Stocke et al. 1991). This diagram provides an idea on whether a galaxy may host an active nucleus. This is because AGN have enhanced X-ray emission for a given optical 
A\&A 552, A135 (2013)
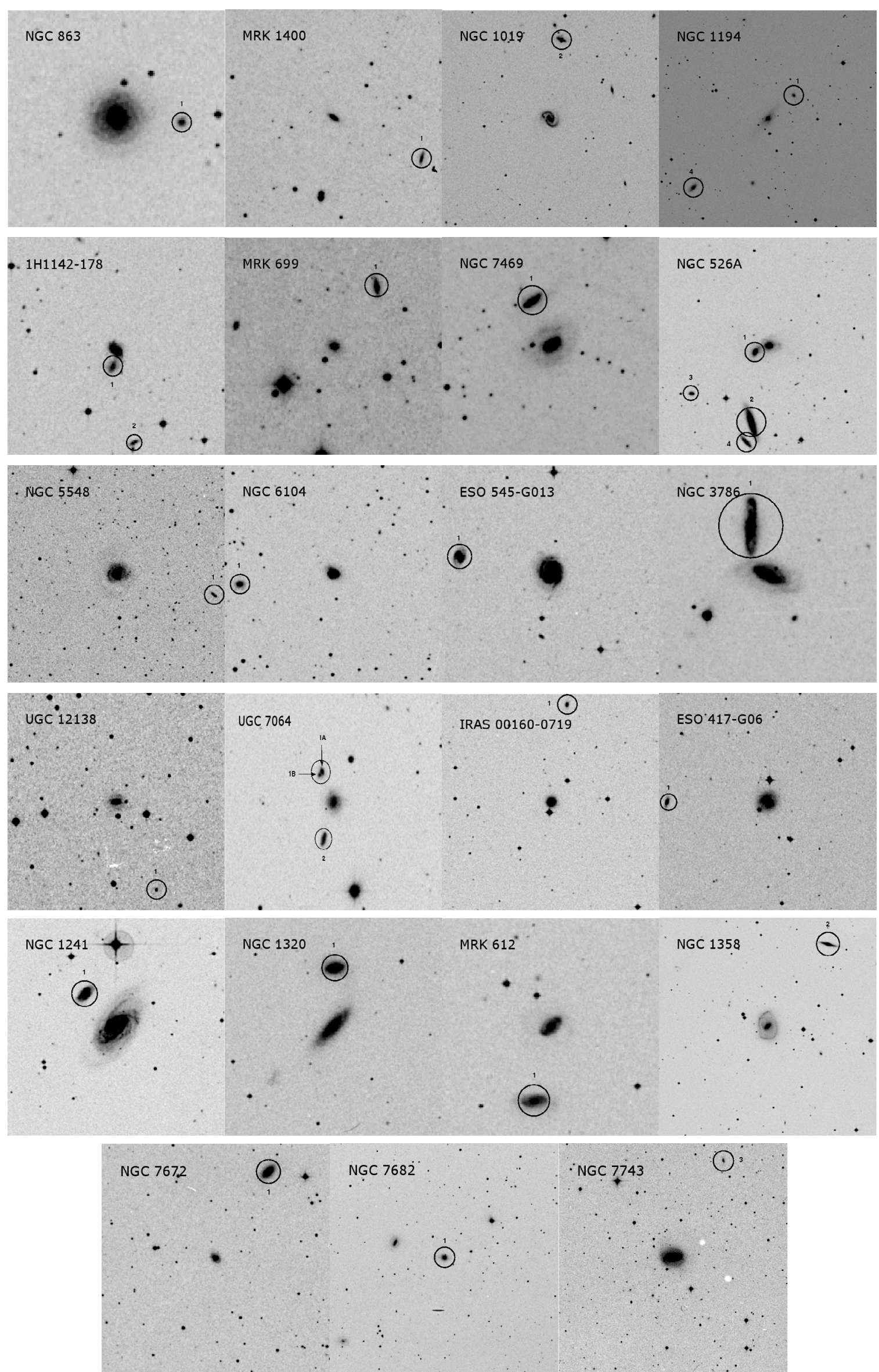

Fig. 3. Images of the AGN galaxies and their neighbours. The AGN is located in the centre of the image except from NGC 7682, which is easily spotted on the left of the image. 
magnitude relative to ALG galaxies. The space usually populated by AGN is shown between the continuous lines. The central Seyfert galaxies are shown as filled points, but since X-ray flux has not been corrected for X-ray absorption, a number of absorbed AGN galaxies lie between the lower continuous line and the dashed line, while the heavily absorbed Sy2 NGC 7743 (Akylas \& Georgantopoulos 2009), lie far below the dashed line. One neighbour that lies in the AGN regime (NGC 7682-N1) can be clearly seen. This has been classified as a TO galaxy in the optical spectroscopic analysis and is one of the three neighbours (for which XMM-Newton observations are available, see Table 4) having an active nucleus based on optical spectroscopy. Additional information on the nature of our sources can be extracted from the hardness ratios. Two sources NGC 526-N2 and NGC 1358-N2 have hardness ratios suggesting an absorption of $N_{\mathrm{H}} \approx 10^{22} \mathrm{~cm}^{-2}$, consistent with the presence of a moderately obscured active nucleus. Both these galaxies present no optical emission lines and thus are classified as ALG, based on their optical spectra. In other words, the lack of optical emission lines from the nucleus of these objects could be a result of obscuration and indeed this seems to be the case, since the detection limit of the EW of emission lines is low enough. In addition, we should mention here that all galaxies among those that fall in fields observed by XMM-Newton, classified as ALG through optical spectroscopy, present X-ray emission. In contrast, all SF galaxies except one in the X-ray subsample do not show an X-ray detection.

We should note here that unobscured low accretion rate Sy2 objects and/or low luminosity AGN, where the narrow line region (NLR) cannot be detected by means of optical spectroscopy, or even X-ray binaries may account for the X-ray detection of unobscured ALG galaxies. However, emission from $\mathrm{X}$-ray binaries is not detected in the spectra of the SFNs rendering this interpretation less plausible. This analysis therefore implies that the total fraction of neighbours of AGN that show recent star formation or AGN, based on optical spectroscopy or $\mathrm{X}$-ray observations, is at least $80 \%$ and possibly quite higher. This matter will be fully addressed in future work.

\section{Discussion and conclusions}

We have investigated the close environment $\left(\leq 100 h^{-1} \mathrm{kpc}\right)$ of a local sample $(z<0.034)$ of AGN. In particular we explored the spectroscopic, photometric and X-ray properties of 30 neighbouring galaxies around 10 Sy 1 and 13 Sy2 galaxies. Based on optical spectroscopy, we found that the large majority of these neighbours show some activity, mostly recent star formation (emission line spectrum) but AGN as well. In addition, our X-ray analysis of a subsample of neighbours with public XMM-Newton observations showed that the neighbours that are classified as ALG based on optical spectroscopy might have a low-luminosity active core, since all of them are X-ray detected, while two out of five appear to have a moderately obscured active nucleus. The X-ray detections could be due to X-ray binaries, but we argue that this is less probable since the pure star-forming neighbours do not show any X-ray emission down to the flux limit of the available observations. From both optical spectroscopy and X-ray observations, it becomes clear that the fraction of AGN's neighbours that exhibit recent star formation and/or nuclear activity, within $100 \mathrm{~h}^{-1} \mathrm{Mpc}$, is $>80 \%$ and possibly higher.

Furthermore, the close neighbours of Sy1 galaxies, especially the SFNs, are less ionized and have lower values of $\mathrm{EW}(\mathrm{H} \alpha)$ than do those of Sy2 and thus seem to be a different, more evolved population than those of Sy2s. Other discovered trends in metallicity, host galaxy size, and age of the most recent starburst event indicate possible physical differences between the neighbours of Sy1 and Sy2 galaxies as well, which may link AGN activity with interactions.

Indeed, over the past two decades there have been several studies that supported the idea of an evolutionary sequence from starburst to Seyfert galaxies (e.g. Storchi-Bergmann et al. 2001, see also Introduction). Furthermore, there are also studies that separate Type I from Type II objects (e.g. Hunt et al. 1997; Maiolino et al. 1997; Gu et al. 2001), implying that recent star formation is only present in Type II objects (see also Coldwell et al. 2009). Based on the number and proximity of close $\left(\$ 60-100 h^{-1} \mathrm{kpc}\right)$ neighbours, around different types of active (Sy1, Sy2 and BIRG) galaxies (e.g. Dultzin-Hacyan et al. 1999; Krongold et al. 2002; Koulouridis et al. 2006a,b), a very interesting evolutionary sequence has been suggested, starting with a close interaction that triggers the formation of a nuclear starburst, subsequently evolving to a Type 2 Seyfert, and finally to a Sy1. Recent observational results by Villarroel et al. (2012) and Kollatschny et al. (2012) also seem to support this scheme. This sequence is likely independent of luminosity, since similar trends have been proposed for LINERs (Krongold et al. 2003) and ULIRGs and quasars (Fiore et al. 2008 and references therein). The above findings were also supported by numerical simulations (Hopkins et al. 2008), which outlined such an evolutionary scheme for merging galaxies. The proposed activity evolution can explain the excess of starbursts and Type 2 AGN in interacting systems, as well as the lack of Type 1 AGN in compact groups of galaxies (Martínez et al. 2008) and galaxy pairs (e.g., Gonzalez et al. 2008).

Since the physical properties of the neighbours should be reflected in the state of the central active galaxy, we argue that our results may be going in the same direction as those of our previous papers (Koulouridis et al. 2006a,b), supporting an evolutionary sequence of galaxy activity, driven by interactions, the main path of which follows the sequence of induced star formation, Sy2 and finally Sy1 phase. A time delay should exist between the pure star-forming and AGN phases (see discussion in the Introduction), where active nucleus and circumnuclear starburst coexist. In this initial phase, the nucleus is heavily obscured by the still star-forming molecular clouds, and it can be observed as a transition stage of composite Sy2-starburst objects. We should note here that according to Ballantyne et al. (2006), a non-evolving torus cannot provide the AGN obscuration over all cosmic time and that extra obscuration by star formation is needed.

The most probable manner for the AGN to dominate is to eliminate the starburst, possibly by the AGN outflows or by radiation pressure. We point out that a great theoretical success of the starburst/AGN connection is the quenching of the induced star formation by the AGN feedback, which can explain the formation of red and dead elliptical galaxies (e.g. Springel et al. 2005a; Di Matteo et al. 2005; Khalatyan et al. 2008). This can be achieved by outflows from the core that have enough energy to dissipate the material around it and thus suffocate star formation (e.g. Krongold et al. 2007, 2009; Blustin et al. 2008; Hopkins \& Elvis 2010; Novak et al. 2011; Cano-Díaz et al. 2012; Zubovas \& King 2012). Recent observational studies and simulations have shown that ionized outflows from the AGN may carry enough energy to cease star formation in the host galaxy rapidly, in less than 1 Gyr (see for example Kaviraj et al. 2011). As the starburst fades (see relevant discussion and references in the Introduction), the Seyfert 2 state starts dominating, to be 
followed at the end by a totally unobscured Sy1 state, plausibly $\sim 1$ Gyr after the initial interaction (see Krongold et al. 2002). More details about the co-evolution of the torus and the AGN are given by Liu \& Zhang (2011), supporting our evolutionary scheme. We should note here that recent observations (Hasinger et al. 2008; Treister et al. 2010) verified a significant increase in the Type 2 AGN fraction with redshift, a fact that agrees with our evolutionary scheme.

Alternatively, there is a possibility that the SFN neighbours of Sy1 galaxies are systematically more massive compared to those of Sy2 and that their older stellar population is due to downsizing; i.e., more massive galaxies have evolved earlier, while less massive ones exhibit more recent star formation and thus a younger stellar population. However, there is no obvious explanation on why more massive galaxies should be located preferentially near Sy1 galaxies and not Sy2. The combination of both downsizing and the interaction driven sequence, as presented previously, can also be at work.

We stress that the suggested evolutionary scenario does not completely invalidate the unification scheme. It implies that the orientation of the torus can determine the AGN phenomenology only at specific phases of the evolutionary sequence. In particular, this probably occurs when the obscuring molecular clouds form the torus (possibly when the AGN activity reaches its peak $\sim 0.5$ Gyr after the initial interactions (Kaviraj et al. 2011) and before being completely swept away, possibly after 1 Gyr (Krongold et al. 2002). From our point of view, in an ever-evolving universe an evolutionary scheme is more probable than the original unification paradigm, which proposes a rather static view of AGN. Of course, orientation could and should also play a role between the obscured Sy 2 and Sy1 phase, when the relaxing obscuring material forms a toroidal structure.

There are still many unresolved issues and caveats concerning these suggestions, since the evolutionary sequence is not unique and should also depend on the geometry, the density, and other factors of the obscuring and the accreting material, as well as on the mass of the host galaxy and its black hole. Furthermore, the sample presented in this pilot study is rather small, and the results should be considered as indicative and should be confirmed by analysis of larger samples.

Acknowledgements. E.K. thanks the IUNAM and INAOE, where a major part of this work was completed, for their warm hospitality. We also thank the OAGH and OAN-SPM staff for excellent assistance and technical support at the telescopes. V.C. acknowledges funding by CONACyT research grants 54480 and 15149 (México). MP acknowledges funding by the Mexican Government research grant No. CONACyT 49878-F and DD support from grant PAPIIT IN111610 from DGAPA, UNAM. Y.K. acknowledges support from CONACyT 168519 grant and UNAM-DGAPA PAPIIT IN103712 grant. This research has made use of the USNO-B catalog (Monet et al. 2003) and the MAPS Catalog of POSS I (Cabanela et al. 2003) supported by the University of Minnesota (the APS databases can be accessed at http://aps.umn.edu/). The STARLIGHT project is supported by the Brazilian agencies CNPq, CAPES and FAPESP and by the FranceBrazil CAPES/Cofecub programme. Funding for the SDSS and SDSS-II has been provided by the Alfred P. Sloan Foundation, the Participating Institutions, the National Science Foundation, the US Department of Energy, the National Aeronautics and Space Administration, the Japanese Monbukagakusho, the Max Planck Society, and the Higher Education Funding Council for England. The SDSS web site is http://www.sdss.org/. Finally, we would like to thank the anonymous referee for his or her comments and suggestions that helped to significantly improve our paper.

\section{References}

Akylas, A., \& Georgantopoulos, I. 2009, A\&A, 500, 999

Antonucci, R. 1993, ARA\&A, 31, 473

Asari, N. V., Cid Fernandes, R., Stasińska, G., et al. 2007, MNRAS, 381, 263
Ballantyne, D. R., Everett, J. E., \& Murray, N. 2006, ApJ, 639, 740 Baldwin, J. A., Phillips, M. M., \& Terlevich, R. 1981, PASP, 93, 5 Blustin, A. J., Dwelly, T., Page, M. J., et al. 2008, MNRAS, 390, 1229 Boisson, C., Joly, M., Moultaka, J., Pelat, D., \& Serote Roos, M. 2000, A\&A 357,850

Boisson, C., Joly, M., Pelat, D., \& Ward, M. J. 2004, A\&A, 428, 373

Cabanela, J. E., Humphreys, R. M., Aldering, G., et al. 2003, PASP, 115, 837

Cano-Díaz, M., Maiolino, R., Marconi, A., et al. 2012, A\&A, 537, L8

Carrera, F. J., Ebrero, J., Mateos, S., et al. 2007, A\&A, 469, 27

Cid Fernandes, R. J., Storchi-Bergmann, T., \& Schmitt, H. R. 1998, MNRAS, 297, 579

Cid Fernandes, R., Heckman, T., Schmitt, H., Delgado, R. M. G., \& Storchi-Bergmann, T. 2001, ApJ, 558, 81

Cid Fernandes, R., Gu, Q., Melnick, J., et al. 2004, MNRAS, 355, 273

Cid Fernandes, R., González Delgado, R. M., Storchi-Bergmann, T., Martins, L. P., \& Schmitt, H. 2005, MNRAS, 356, 270

Cid Fernandes, R., Asari, N. V., Sodré, L., et al. 2007, MNRAS, 375, L16

Cisternas, M., Jahnke, K., Inskip, K. J., et al. 2011, ApJ, 726, 57

Coldwell, G. V., Lambas, D. G., Söchting, I. K., \& Gurovich, S. 2009, MNRAS, 399,88

Davies, R., Genzel, R., Tacconi, L., Mueller Sánchez, F., \& Sternberg, A. 2007, in The Central Engine of Active Galactic Nuclei, ASP Conf. Ser., 373, 639

Davies, R., Burtscher, L., Dodds-Eden, K., \& Orban de Xivry, G. 2012, J. Phys. Conf. Ser., 372, 12046

Di Matteo, T., Springel, V., \& Hernquist, L. 2005, Nature, 433, 604

Dultzin-Hacyan, D., \& Benitez, E. 1994, A\&A, 291, 720

Dultzin-Hacyan, D., Krongold, Y., Fuentes-Guridi, I., \& Marziani, P. 1999, ApJ, 513, L111

Ellison, S. L., Patton, D. R., Simard, L., \& McConnachie, A. W. 2008, AJ, 135, 1877

Ellison, S. L., Patton, D. R., Mendel, J. T., \& Scudder, J. M. 2011, MNRAS, 418, 2043

Fiore, F., Grazian, A., Santini, P., et al. 2008, ApJ, 672, 94

Galaz, G., Herrera-Camus, R., Garcia-Lambas, D., \& Padilla, N. 2011, ApJ, 728, 74

Georgakakis, A., Coil, A. L., Laird, E. S., et al. 2009, MNRAS, 397, 623

González, J. J., Krongold, Y., Dultzin, D., et al. 2008, Rev. Mex. Astron. Astrofis. Conf. Ser., 32, 170

Gu, Q., Maiolino, R., \& Dultzin-Hacyan, D. 2001, A\&A, 366, 765

Hasinger, G. 2008, A\&A, 490, 905

Ho, L. C. 2008, ARA\&A, 46, 475

Ho, L. C., Filippenko, A. V., \& Sargent, W. L. W. 1997, ApJS, 112, 315

Hopkins, P. F., \& Elvis, M. 2010, MNRAS, 401, 7

Hopkins, P. F., Hernquist, L., Cox, T. J., \& Kereš, D. 2008, ApJS, 175, 356

Hunt, L. K., Malkan, M. A., Salvati, M., et al. 1997, ApJS, 108, 229

Ideue, Y., Taniguchi, Y., Nagao, T., et al. 2012, ApJ, 747, 42

Kauffmann, G., Heckman, T. M., Tremonti, C., et al. 2003, MNRAS, 346, 1055

Kaviraj, S., Schawinski, K., Silk, J., \& Shabala, S. S. 2011, MNRAS, 415, 3798

Kawakatu, N., Anabuki, N., Nagao, T., Umemura, M., \& Nakagawa, T. 2006, ApJ, 637, 104

Kewley, L. J., Heisler, C. A., Dopita, M. A., \& Lumsden, S. 2001, ApJS, 132, 37

Kewley, L. J., Geller, M. J., \& Barton, E. J. 2006a, AJ, 131, 2004

Kewley, L. J., Groves, B., Kauffmann, G., \& Heckman, T. 2006b, MNRAS, 372, 961

Khalatyan, A., Cattaneo, A., Schramm, M., et al. 2008, MNRAS, 387, 13

Knapen, J. H., \& James, P. A. 2009, ApJ, 698, 1437

Kollatschny, W., Reichstein, A., \& Zetzl, M. 2012, A\&A, 548, A37

Kormendy, J., \& Richstone, D. 1995, ARA\&A, 33, 581

Krongold, Y., Dultzin-Hacyan, D., \& Marziani, P. 2002, ApJ, 572, 169

Krongold, Y., Nicastro, F., Brickhouse, N. S., et al. 2003, ApJ, 597, 832

Krongold, Y., Nicastro, F., Elvis, M., et al. 2007, ApJ, 659, 1022

Krongold, Y., Jiménez-Bailón, E., Santos-Lleo, M., et al. 2009, ApJ, 690, 773

Koulouridis, E., Plionis, M., Chavushyan, V., et al. 2006a, ApJ, 639, 37

Koulouridis, E., Chavushyan, V., Plionis, M., Krongold, Y., \& Dultzin-Hacyan, D. 2006b, ApJ, 651, 93

León-Tavares, J., Valtaoja, E., Chavushyan, V. H., et al. 2011a, MNRAS, 411, 1127

León-Tavares, J., Valtaoja, E., Tornikoski, M., Lähteenmäki, A., \& Nieppola, E. 2011b, A\&A, 532, A146

Li, C., Kauffmann, G., Heckman, T. M., White, S. D. M., \& Jing, Y. P. 2008, MNRAS, 385, 1915

Lipovetskij, V. A., Neizvestnyj, S. I., \& Neizvestnaya, O. M. 1987, in Soobshcheniya Spetsial'noj Astrofizicheskoj Observatorii, 55

Liu, Y., \& Zhang, S. N. 2011, ApJ, 728, L44

Lynden-Bell, D. 1969, Nature, 223, 690

Magorrian, J., Tremaine, S., Richstone, D., et al. 1998, AJ, 115, 2285 
E. Koulouridis et al.: Neighbours of Seyfert galaxies

Maiolino, R., \& Rieke, G. H. 1995, ApJ, 454, 95

Maiolino, R., Ruiz, M., Rieke, G. H., \& Papadopoulos, P. 1997, ApJ, 485, 552

Marquez, I., \& Moles, M. 1994, AJ, 108, 90

Martínez, M. A., Del Olmo, A., Coziol, R., \& Perea, J. 2008, Rev. Mex. Astron. Astrofis. Conf. Ser., 32, 164

Mateus, A., Sodré, L., Cid Fernandes, R., et al. 2006, MNRAS, 370, 721

Melia, F., \& Falcke, H. 2001, ARA\&A, 39, 309

Moles, M., Marquez, I., \& Perez, E. 1995, ApJ, 438, 604

Monet, D. G., Levine, S. E., Canzian, B., et al. 2003, AJ, 125, 984

Müller Sánchez, F., Davies, R. I., Genzel, R., et al. 2008, Rev. Mex. Astron. Astrofis. Conf. Ser., 32, 109

Nelson, C. H., \& Whittle, M. 1996, ApJ, 465, 96

Novak, G. S., Ostriker, J. P., \& Ciotti, L. 2011, ApJ, 737, 26

Silverman, J. D., Kampczyk, P., Jahnke, K., et al. 2011, ApJ, 743, 2

Springel, V., Di Matteo, T., \& Hernquist, L. 2005, ApJ, 620, L79

Stasińska, G., Cid Fernandes, R., Mateus, A., Sodré, L., \& Asari, N. V. 2006, MNRAS, 371, 972
Stocke, J. T., Morris, S. L., Gioia, I. M., et al. 1991, ApJS, 76, 813 Storchi-Bergmann, T., González Delgado, R. M., Schmitt, H. R., Cid Fernandes, R., \& Heckman, T. 2001, ApJ, 559, 147

Tang, Y.-W., Kuo, C.-Y., Lim, J., \& Ho, P. T. P. 2008, ApJ, 679, 1094

Treister, E., Urry, C. M., Schawinski, K., Cardamone, C. N., \& Sanders, D. B. 2010, ApJ, 722, L238

Tresse, L., Maddox, S., Loveday, J., \& Singleton, C. 1999, MNRAS, 310, 262

Umemura, M., Fukue, J., \& Mineshige, S. 1998, MNRAS, 299, 1123

Veilleux, S., \& Osterbrock, D. E. 1987, ApJS, 63, 295

Villarroel, B., Korn, A., \& Matsuoka, Y. 2012 [arXiv: 1211.0528]

Villforth, C., Sarajedini, V., \& Koekemoer, A. 2012, MNRAS, 426, 360

Volonteri, M., Haardt, F., \& Gültekin, K. 2008, MNRAS, 384, 1387

Wake, D. A., Miller, C. J., Di Matteo, T., et al. 2004, ApJ, 610, L85

Wang, L., \& Kauffmann, G. 2008, MNRAS, 391, 785

Watson, M. G., Schröder, A. C., Fyfe, D., et al. 2009, A\&A, 493, 339

Wild, V., Heckman, T., \& Charlot, S. 2010, MNRAS, 405, 933

Zubovas, K., \& King, A. 2012, ApJ, 745, L34 


\section{Appendix A}
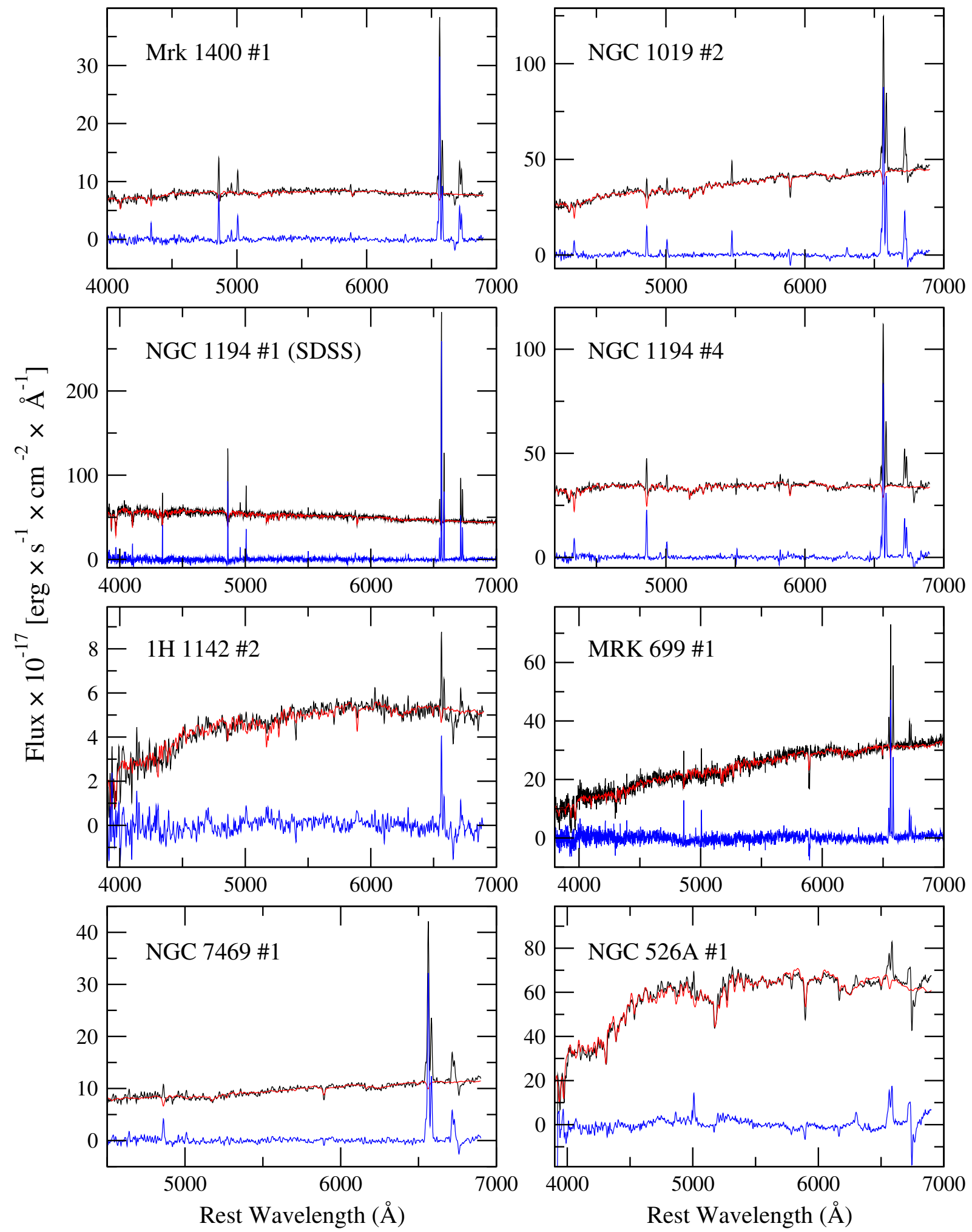

Fig. A.1. Spectra of the ELG neighbours of AGN galaxies, listed in Tables 1 and 2. 
E. Koulouridis et al.: Neighbours of Seyfert galaxies
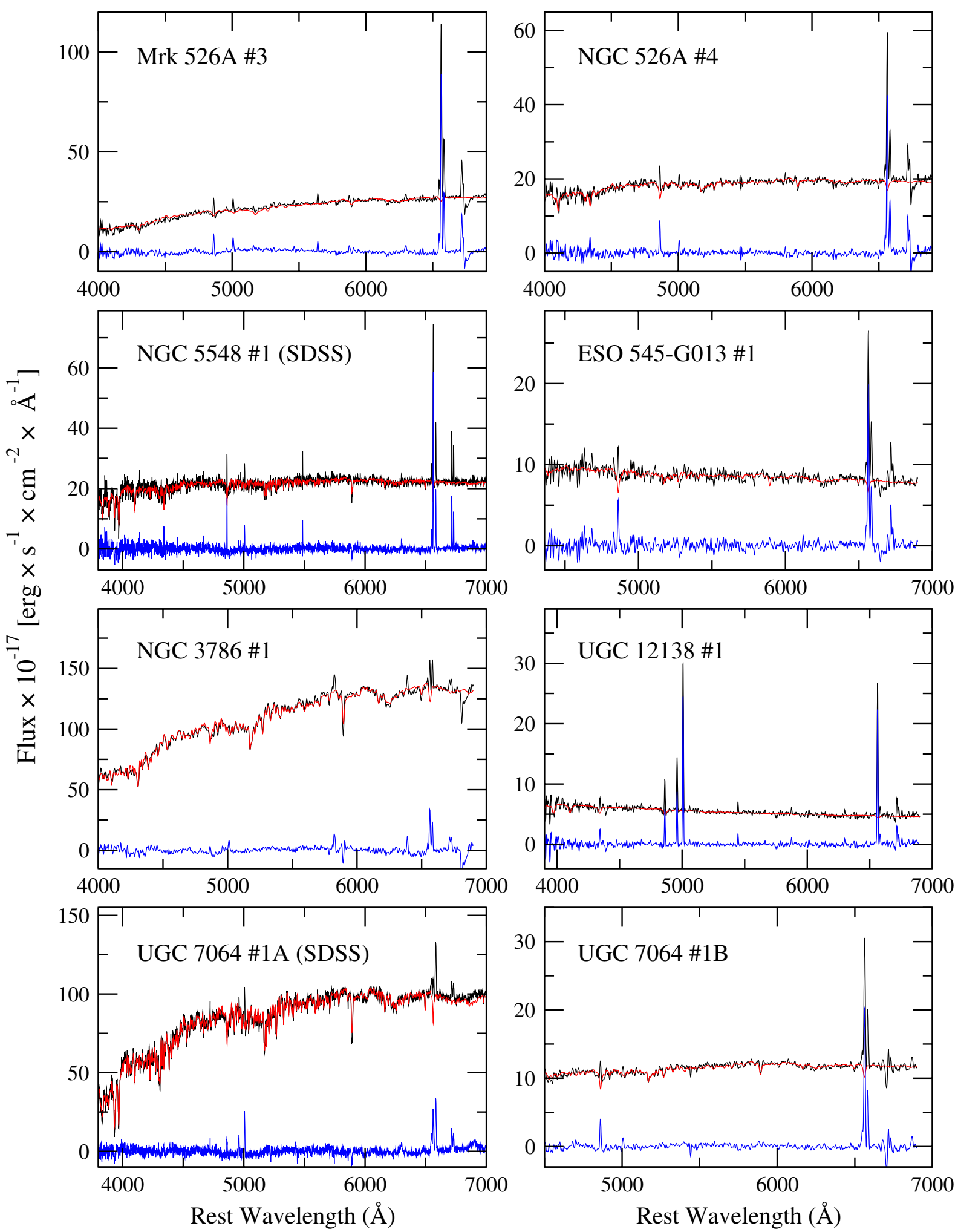

Fig. A.1. continued. 

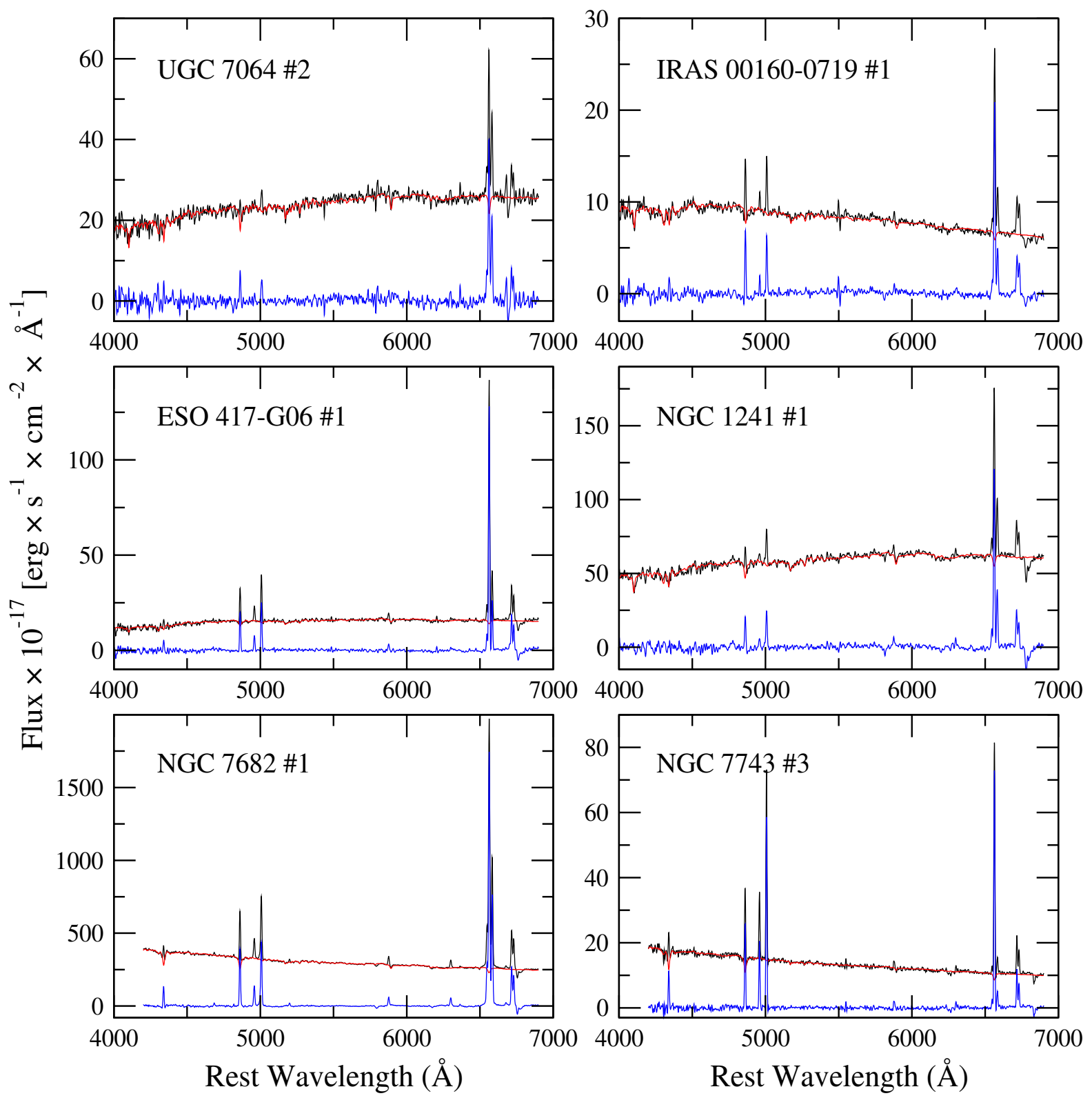

Fig. A.1. continued.

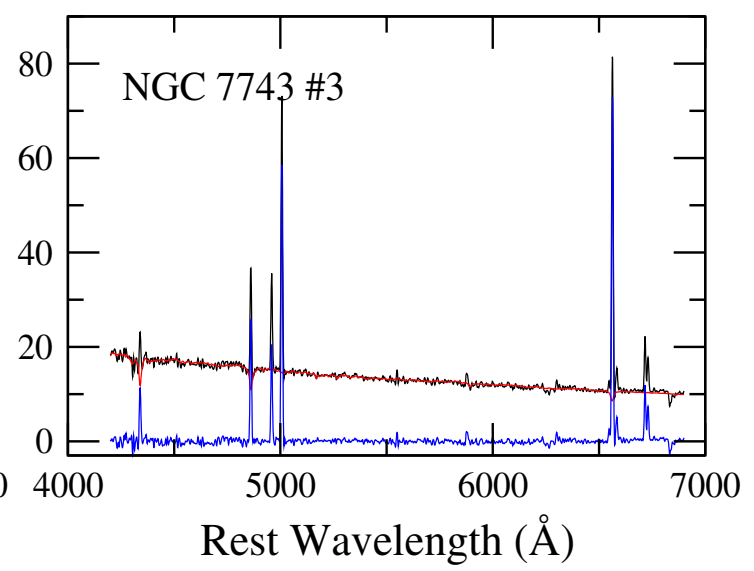

